EGG-RAAM-11086

\title{
Collecting \\ Operational Event Data for Statistical Analysis
}

Corwin L. Atwood

Published September 1994

Idaho National Engineering Laboratory

EG\&G Idaho, Inc.

Idaho Falls, Idaho 83415

Prepared for the

Reliability and Risk Analysis Branch

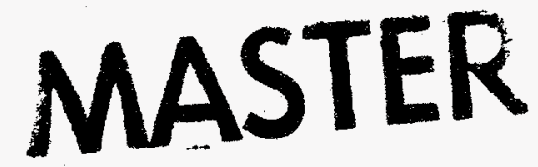

Safety Programs Division

Office for Analysis and Evaluation of Operational Data

U.S. Nuclear Regulatory Commission

Washington, DC 20555

Under DOE Idaho Operations Office

Contract DE-AC07-76ID01570

FIN E8205 


\section{DISCLAIMER}

This report was prepared as an account of work sponsored by an agency of the United States Government. Neither the United States Government nor any agency thereof, nor any of their employees, make any warranty, express or implied, or assumes any legal liability or responsibility for the accuracy, completeness, or usefulness of any information, apparatus, product, or process disclosed, or represents that its use would not infringe privately owned rights. Reference herein to any specific commercial product, process, or service by trade name, trademark, manufacturer, or otherwise does not necessarily constitute or imply its endorsement, recommendation, or favoring by the United States Government or any agency thereof. The views and opinions of authors expressed herein do not necessarily state or reflect those of the United States Government or any agency thereof. 


\section{DISCLAIMER}

Portions of this document may be illegible in electronic image products. Images are produced from the best available original document. 


\section{ABSTRACT}

This report gives guidance for collecting operational data to be used for statistical analysis, especially analysis of event counts. It discusses how to define the purpose of the study, the unit (system, component, etc.) to be studied, events to be counted, and demand or exposure time. Examples are given of classification systems for events in the data sources. A checklist summarizes the essential steps in data collection for statistical analysis. 


\section{SUMMARY}

This report gives guidance for collecting operational data to be used for statistical analysis, especially analysis of event counts.

It is essential to have explicit definitions for the following:

- $\quad$ Purpose(s) of the study

- Boundaries of the unit (system, component, etc.) to be studied

- Events to be counted, called "hits," typically some kinds of failure

- Demand or exposure time.

When defining hits, the study team must develop a classification system for describing the events that occurred. Topics considered include classification of command faults, failures when there was no accompanying demand, partial failures, and several failures in immediate succession. Examples are given of failure-mode classifications used in other studies.

Some techniques are given to help ensure that the hits are all found and are correctly coded. Estimation of the demands or exposure time is discussed. The report closes with a checklist of items to be completed in a data collection effort, when the data are to be used for statistical analysis. The topics in the checklist are

1. What is the purpose of the study?

2. What is the unit (system, component, etc.) being studied?

3. What are the best sources of data?

4. How will the number of demands or the exposure time be determined?

5. How can the hits (typically, failures of the system) all be found? 
6. How should all the events in the data source(s) be classified?

7. Reflect and review.

8. Complete the data collection. 


\section{FOREWORD}

The Office for Analysis and Evaluation of Operational Data (AEOD) of the U.S. Nuclear Regulatory Commission (NRC) is continually reviewing data from U.S. commercial nuclear power plants. Statistical analysis forms an important part of this work. Accordingly, the Reliability and Risk Analysis Branch of AEOD has asked the Idaho National Engineering Laboratory (INEL) to write a series of reports presenting the appropriate statistical tools for the kinds of data most commonly encountered. These reports are being written in parallel with a series of studies on the performance of safety systems in nuclear power plants, and reflect the influence of those studies.

The reports are expected to provide tools and guidance to analysts of NRC data, although the statistical methods can, by their nature, be applied to data from many other fields. The reports are intended to quickly help a new user. For example, the reports on statistical methods should be readable and immediately usable by a person with training in statistics but with no experience analyzing such data. The report on collecting operational data should be understandable by anyone with a technical background, although the examples are slanted towards an engineer with nuclear experience. In addition, the reports should be usable as texts or references in short courses for persons with less training.

The first three reports in this series, appearing in September, 1994, are

Collecting Operational Event Data for Statistical Analysis, EGG-RAAM-11086, by Corwin L. Atwood

Hits per Trial: Basic Analysis of Binomial Data, EGG-RAAM-11041, by Corwin L. Atwood

Events in Time: Basic Analysis of Poisson Data, EGG-RAAM-11088, by M. E. Engelhardt

Other reports in the series are planned for 1995. 


\section{ACKNOWLEDGMENTS}

Cindy Gentillon, Gary Grant, Harry Martz, and Marty Sattison provided very helpful review comments. 


\section{CONTENTS}

ABSTRACT $\ldots \ldots \ldots \ldots \ldots \ldots \ldots \ldots \ldots \ldots \ldots \ldots \ldots \ldots \ldots \ldots$

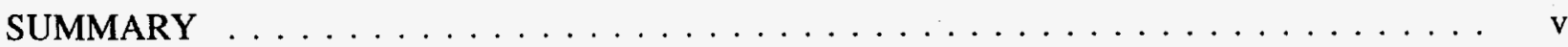

FOREWORD $\ldots \ldots \ldots \ldots \ldots \ldots \ldots \ldots \ldots \ldots \ldots \ldots \ldots \ldots \ldots$

ACKNOWLEDGMENTS $\ldots \ldots \ldots \ldots \ldots \ldots \ldots \ldots \ldots \ldots \ldots \ldots$ ix

INTRODUCTION $\ldots \ldots \ldots \ldots \ldots \ldots \ldots \ldots \ldots \ldots \ldots \ldots \ldots$

DEFINITIONS $\ldots \ldots \ldots \ldots \ldots \ldots \ldots \ldots \ldots \ldots \ldots \ldots \ldots \ldots$

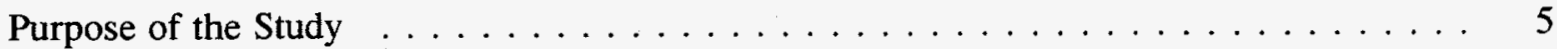

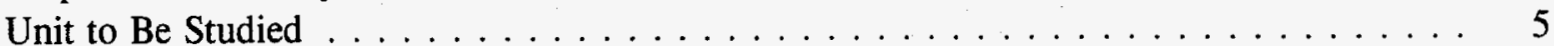

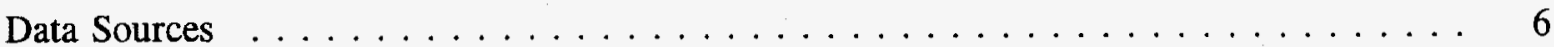

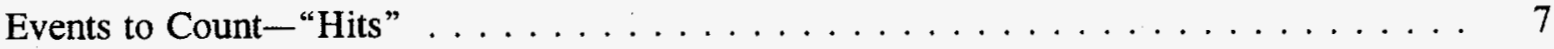

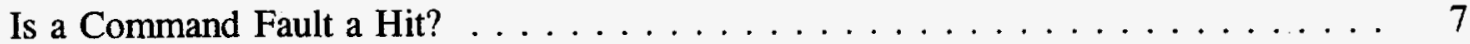

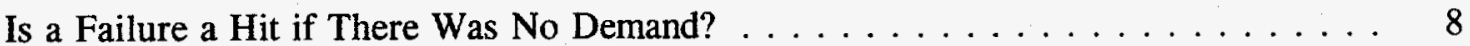

Is a Partial Failure a Hit? $\ldots \ldots \ldots \ldots \ldots \ldots \ldots$

Are Several Failures in Immediate Succession Separate Hits? . . . . . . . . . . . . 9

Which Failure Modes Are Hits? . . . . . . . . . . . . . . . . . . . 10

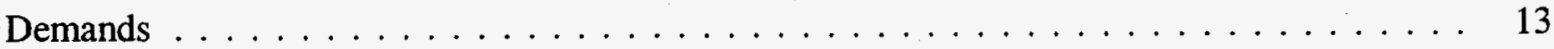

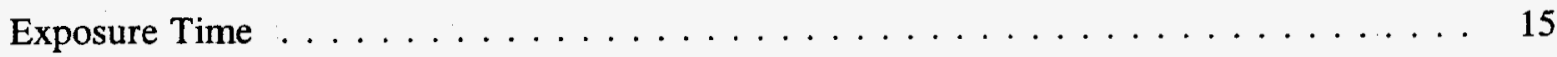

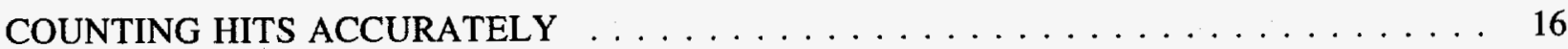

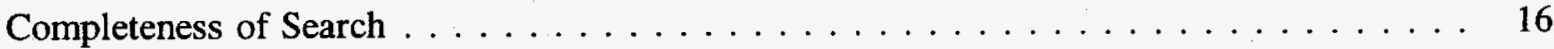

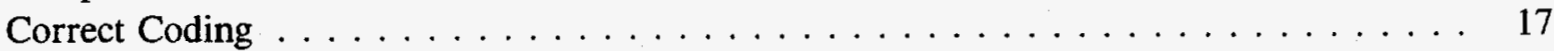

COUNTING OR ESTIMATING DEMANDS OR EXPOSURE TIME $\ldots \ldots \ldots \ldots$

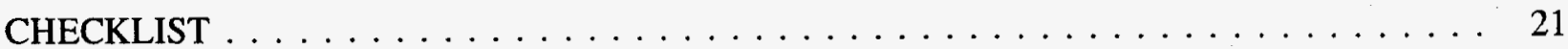

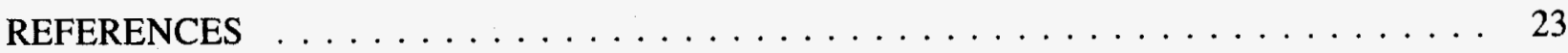

TABLES

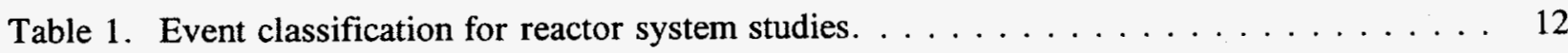

Table 2. Event classification for study of common cause failures. . . . . . . . . . . 13 


\section{Collecting Operational Event Data for Statistical Analysis}

\section{INTRODUCTION}

This report gives guidance for collecting operational data to be used in statistical analysis. Operational data means data collected from a process whose primary purpose is not merely the generation of the data. Data from power generation at a nuclear power plant or data from a chemical manufacturing activity are examples. Such a process is distinguished from a laboratory test or experiment, which is planned and performed solely to give the desired data. Portions of operational data, such as monthly surveillance tests, may indeed consist of planned tests. Nevertheless, operational data tend to be messier than data from a well-designed experiment. Unexpected kinds of failures, such as human errors, appear in operational data; these might be discarded in a report of a sequence of laboratory experiments. Operational data are nearly always unbalanced, in that the operating times or the numbers of trials vary between data sources. Operational data reflect a wider range of conditions than do data from any single set of tests or experiments. Operational data may report facts that the data collector will deliberately ignore, and at the same time fail to report certain vital pieces of information.

Many of the considerations given in this report, such as the need for careful definitions, are also important in planning an experiment. Moreover, a data analyst, considering a completed data set, must be aware of the many judgments that influenced the data set, an issue that Mosleh (1986) illuminates. The viewpoint here, however, is of neither a planner nor an analyst, but of someone sorting through operational records to extract useful data.

The emphasis is on discrete events, typically failure events, and the statistical analysis often involves estimating event probabilities or rates. Some of the suggestions in this report, however, also apply to other types of records and analyses.

Normally, a person collecting operational data is experienced with how the systems work, but is not necessarily trained in statistical methods. Similarly, a person doing the statistical analysis may be inexperienced with the systems' functions and possible failures. For brevity, the first kind of person is referred to here as an "engineer" or a "data collector," and the second kind of person is referred to as a "statistician" or "statistical analyst." Although one person may be well grounded in both disciplines, this is unusual. This report is directed towards both kinds of people, but especially towards data collectors. 
While perusing the event records, a data collector may find it useful to record many kinds of information, perhaps including anecdotes about unexpected kinds of failures, or problems discovered during design reviews. This report, however, only considers data sets that are to be used for statistical analysis, subsets of all the data that might profitably be collected. Here are some examples of the type of analysis that might be intended.

- Estimate the probability of failure on demand, for a standby component or system, and quantify the uncertainty in the estimate. The data set contains a count of the number of failures and a corresponding number of demands. A commonly used estimate of failure probability is by the fraction (number of failures)/(number of demands). It is a dimensionless quantity. The uncertainty in the estimate depends on how many demands and failures were counted; the larger the data set, the smaller the uncertainty in the estimate.

- Estimate the rate, at which failures occur in time, for some system or component. In contrast to the example of failures on demand, the data set for this analysis contains a count of failures in some study time. A common estimate of failure rate is the fraction (number of failures)/ (total time when such failures could occur). It has dimension $1 /$ time. The time when failures could occur is called exposure time in this report; it may be different from clock time. The uncertainty depends on the failure count and the exposure time, with a long exposure time reducing the uncertainty in the estimate.

- Make a comparison between failure rates or between failure probabilities, such as:

- Do the failure rates or probabilities change over time, for example from year to year?

- Are the failure rates or probabilities the same at all plants in the study?

- Are the failure rates or probabilities the same for like components in different systems?

- Are the failure probabilities the same on different kinds of demands, such as unplanned demands and various surveillance tests?

- Are the failure rates or probabilities the same for different kinds of failures?

- In different groups of plants, is the fraction of failures that are caused by personnel error the same or different? 
The list of things to compare can be extended indefinitely, but in every case we are estimating a rate or probability in two or more data sources, and comparing the estimates, accounting statistically for the uncertainty in the estimates.

Although many such statistical analyses could be attempted, the data requirements are very similar: the data must give

(1) the number of failures that occurred, and

(2) the corresponding number of demands or the exposure time.

Point (2) is worth emphasizing. People involved in the operational process-engineers, maintenance personnel, supervisors-give most of their attention to failures and possible failures. Therefore, operational records usually contain more information about past failures than about past successes. This information about the failures can provide useful insights into the qualitative nature of failures. But for quantitative studies, which give failure probabilities or failure rates, the number of failures is not enough; the demand count or the exposure time must also be known or estimated.

There are three practical problems: defining all the terms, counting the failures accurately, and counting or estimating the demands or exposure time. These three issues are the subjects of the three major sections of this report. The section covering definitions is by far the longest section, and may seem to include aspects of the counting itself. However, the section on definitions deals with conceptual issues. As an analogy, if we were sending people out to count pine trees in a forest, we would first deal with the definitions, perhaps even before stepping outside: How do I tell a pine tree from a fir or a spruce? Do I count seedlings? If two trunks grow from one base, is that one tree or two? Only after such questions were answered would we worry about practical techniques of efficient and accurate counting. Similarly, this report first deals with conceptual definitions, and then with the practical aspects of counting failures and counting or estimating demands or exposure time. 


\section{DEFINITIONS}

The primary reason for mistakes in data collection is neglect of the definitions. People say, "Oh, I thought we wanted to . . .," or "Well, I just assumed that . .,", or "No, I've been interpreting this as ..." There are two reasons for neglect of the definitions: The definitions seem obvious, and formulating them carefully is difficult.

A data collector may try to avoid formulating a definition by saying, "I can't define it, but I know it when I see it." This might be defensible if the data collector were the only person involved and the study were very small, but this is almost never the case.

- If the study lasts for weeks or longer, the data collector's ideas may evolve, and writing down the definitions allows the collector to be aware of this and to maintain consistency.

- Several data collectors may work simultaneously (allowing them to check each other), or replace each other over time; they should work consistently.

- The data collectors must communicate well with the other members of the study team, such as the statistical analysts.

- The customer or funder has the right, in fact the responsibility, to know what assumptions were made in the data collection and analysis.

- Later readers of the study report need enough information so that they can decide if the report is relevant for their needs.

For all these reasons, the definitions must be written down and explained.

Therefore, this section lists the things that must be defined, and offers guidance for definitions that

may be relevant for your study. It is highly unlikely that the first set of definitions tried will be adequate. When beginning a new kind of study, plan on a learning period, when the data events reveal inadequacies in the original definitions. There will be several attempts, and long discussions, before really workable definitions have been established. 


\section{Purpose of the Study}

The first thing to define is the purpose of the study. The reason for defining this is that the data to be collected must be relevant for the study. For example, if the study is to estimate the behavior of some portion of a nuclear power plant under design-basis conditions (that is, under some postulated stressful emergency conditions), data from periodic valve tests may not be relevant, because the test conditions do not reflect the design-basis conditions. Alternatively, the valve data may be relevant but of minor importance, because the valves are not the critical elements in plant success under the postulated design-basis event. Or the valve data may be just what is needed. Until the purpose of the study has been defined, no one can say what data to collect.

There may be more than one purpose. If so, different portions of the data may be collected for the different purposes.

A free-wheeling open-ended discussion with the customer is very useful at this point. Some customers may themselves not be sure at first of all the purposes of the proposed study. Others may know all the purposes but may not have verbalized and clearly explained them. The conversation between all parties must continue until the customer's purposes are clear to the data collectors and analysts. The purposes should then be written down, as a final check on whether everyone agrees, and as text that will eventually go into the study report.

The statement of purpose usually includes some limitations, commonly known as scope, schedule, and budget. They are mentioned here as an implicit part of the purpose statement, although they are not emphasized in this report as much as the basic purpose issue, "What do we intend to learn from this study?"

\section{Unit to Be Studied}

Define the unit to be studied. The term unit, or observational unit, is used here to mean the basic thing that is being studied. It could be a type of component, a team of maintenance personnel, a large or small set of hardware elements, or something else. The unit, and its boundaries, must be defined.

The level of detail will depend on the purpose of the study and on the information available. For example, the high pressure coolant injection (HPCI) system consists of a turbine-driven pump, associated valves, and instrumentation and control circuitry. One of these valves can consist of a body, internal piece parts, an operator (such as a motor), and control circuitry. The internal piece parts can vary 
greatly, depending on application. These piece parts, such as the internal pilot valve, may be composed of different materials from the main valve. To exaggerate the point, these materials could in theory be examined at a molecular level. This example illustrates the fact that the study team must decide on the level of detail to be considered. The fundamental element whose properties are being studied is referred to as the observational unit in this report. For failure data, this unit is the fundamental item that can fail.

Sometimes a study requires consideration of more than one observational unit. For example, the automatic depressurization system (ADS) in a boiling water reactor consists of a set of sensors and logic circuitry (the logic control system) and a number of valve trains (valves and their associated operators and circuitry). The logic control system is capable of actuating all the valves. ADS has never been truly demanded in any plant. However, the valves and logic control system are all tested periodically, and the valves are sometimes demanded for functions other than the ADS function. A reasonable approach, therefore, is to collect data separately on the logic control system and on the valve trains. In the nomenclature of this report, there are then two data collection efforts on two observational units, with one kind of unit being the logic control system and the other being a valve train. These units are the fundamental items that can fail, and must be defined carefully at the outset of the study.

In any case, spell out what is included and what is not included in the units under study. For example, if the study concerns performance of a certain type of pump, the pump is what we are calling the unit, and the boundaries of the pump must be established. Does the pump include the motor-driver with its dedicated feeder breaker? Local instrumentation? Remote instrumentation? Obtain or make a diagram if possible. The unit boundaries may be partly determined by the way the available data sources are structured, and it will certainly be affected by the purpose of the study. The definition as written will form an introductory portion of the study report.

\section{Data Sources}

Decide on the best available data sources for the observational unit, consistent with the purpose of the study. Some might question whether identifying data sources belongs in a section dealing with definitions. One justification is that by identifying the best data sources, we are helping define the study. In any case, selection of the data sources comes chronologically at this point in the study, and so is listed here among the things that must be defined.

The data sources must give the relevant events (called "hits" below) that have occurred. The data sources must also give the corresponding number of demands or the corresponding exposure time. 
Several data sources may be identified here, perhaps two that give the failures and one that gives demands. The two that give failures can be used to supplement each other and verify each other, while the one that gives demands may be an entirely different data source. When considering data sources, consider whether they contain the needed information, whether they are complete, whether they are believed to be correct, and whether they are consistent with each other and with the purpose of the study.

\section{Events to Count-“Hits"}

Defining what events to count can be surprisingly complicated. The data sources will probably contain many kinds of events, not all relevant for the statistical analysis. The term "hit" is used here to mean "an event that should be counted." The definition of a hit must be driven by the purpose of the study. If the study has several purposes, the data may need to distinguish between several kinds of hits. To help you define hits, here is a partial list of the questions to ask, along with the reasoning needed to answer them. Every question on the list must be addressed in most studies.

\section{Is a Command Fault a Hit?}

A command fault is an event when the observational unit itself does not need repair, but it does not function as desired because of external inputs or lack of inputs. Examples are

- The unit is a pump. The pump fails to start because the feeder breaker for the motor driver fails (and the pump boundaries have been defined to exclude this feeder breaker).

- The unit is a system of hardware that includes a critical valve. The valve fails to allow flow because it has been manually closed.

- The unit is demanded, but does not respond because it is out of service for maintenance or testing.

The decision of whether to count command faults depends on the purpose of the study. A person in charge of selling, or procuring, pumps would only be interested in how well the pump itself performed, and would therefore not want to count command faults. A person evaluating how well a system performs its function of injecting water would want to count all failures of the pump to perform, including command faults. 
Is a Failure a Hit if There Was No Demand?

Examples of such events are

(1) A severely leaking valve was noticed and was repaired.

(2) During testing, an auxiliary feedwater (AFW) pump failed because it was airbound. As a result, the other two AFW pumps were examined, and one of them was also found to be airbound. Its casing was vented of trapped air before it was tested, and the pump then performed successfully.

(3) After maintenance, a pump's motor-driver circuit breaker was not racked in. This was discovered four days later and corrected, but during the four-day period the pump would not have automatically started on demand. (Assume that command faults are to be counted, and concentrate on the fact that the pump was inoperable but there was no demand.)

(4) A design review discovered that snubbers were missing on a pipe, and therefore the pipe presumably would have failed in an earthquake.

To decide how to treat such cases, refer to the purpose of the study. One possible purpose would involve estimating the probability of failure on demand, and the estimate would be the number of failures on demand divided by the corresponding number of demands. In this case, a failure that occurred or was discovered without an accompanying demand is not a hit. Only the demands that result in failure are hits.

Now suppose instead that the purpose of the study is to estimate the rate at which certain events occur in time. This could be done for two reasons: (a) Some failure modes are caused by age. For example, over time corrosion could cause a valve to stick, whether the valve is operated or not. Therefore, the rate of failures per time is more directly related to the physical process than is the probability of failure on demand, for this failure mechanism. (b) When the number of demands cannot be determined it may be necessary to estimate the rate (with units $1 /$ time) instead of the probability of failure on demand (dimensionless). Time is a surrogate for the unknown demand count.

Assuming, therefore, that the purpose of the study is to estimate the rate per unit of time, consider each of the above examples separately. 
(1) The valve that was discovered leaking is a hit. There are two cases, depending on the function of the valve: (a) The valve was considered as operating when the failure was discovered. (b) The valve was in standby condition, but would not have operated if required because of the leak. In either case, the event is relevant for estimating the rate at which such valves fail in time; it is a hit.

(2) Now consider the second AFW pump, which was vented before it was tested. This event is relevant in estimating the rate (units $1 /$ time) at which pumps fail, and so is a hit. This example involves more than just the second pump, however, and the failure of the two pumps should probably be considered a single common cause event.

(3) The example with the pump's motor-driver circuit breaker is also a hit, because the event is relevant for estimating the rate (units $1 /$ time) that pumps are inoperable. However, one might be concerned about the duration of the event, which is longer than a typical repair time for a pump.

(4) Finally, the case of the missing snubbers is not an event that occurs randomly in time, but a condition that had persisted ever since the plant was built. It could be used to estimate the fraction of plant systems that are not correctly designed for earthquakes, but it should not be used to estimate a failure rate of snubbers, or counted as one pipe failure in so many years.

As can be seen, reasonable people can initially disagree over whether to count a failure that had no corresponding demand. Questionable cases should be discussed by the team of people conducting the study, and not decided by a single data collector.

\section{Is a Partial Failure a Hit?}

Partial failures are events in which the system is degraded, but not completely inoperable. For example, an isolation valve may leak at a certain rate, or a pump may achieve some fraction of the rated flow. How and whether to count them depends on the purpose of the study. The decision should be written into the final study report.

\section{Are Several Failures in Immediate Succession Separate Hits?}

Suppose the system fails to start, is therefore demanded again and fails again, is therefore demanded again and fails again, and so forth. If there is only one problem that caused the repeated failures, many 
analysts would call this a single demand and a single failure. If failure to recover is modeled as a separate failure mode, the event might be modeled as one failure to start, and one failure to recover, with one demand to start and one demand to recover. Two arguments in favor of not counting all the repeats separately are

- If every attempt to start were counted as a separate demand, an operator who continued trying to start the system would make the system look very unreliable-many failures on as many demands-but an operator who did not try to start the system after the first failure would produce only one failure on one demand. In reality, the system is in the same condition each time, and its measured performance should not depend on the stubbornness of the operator.

- Most statistical analyses treat the outcomes of demands as statistically independent, with the probability of failure on one demand independent of what happened on previous demands. When the failures occur in quick succession with no intermediate diagnosis and repair, the failures are dependent, and the usual statistical analysis is not valid.

Therefore, it is common to count a sequence of repeated failures as one failure on one demand. BUT, the decision must always be guided by the purpose of the study, and the decision must be stated in the study report.

\section{Which Failure Modes Are Hits?}

Like everything else, this depends on the purpose of the study. Here is a short case history (Atwood et al. 1993):

The reactor core isolation cooling (RCIC) system is a standby safety system for injecting cooling water into the reactor vessel of a boiling water reactor, as shown in the extremely simplified diagram in Figure 1. The system is used to maintain reactor water level during transient conditions and during accidents if they occur, and it is also demanded for surveillance tests. A study of failure data from the RCIC system was conducted with two purposes: to estimate the probability of system failure on demand under accident conditions, and to gain insights into the types of failures that can occur. The statistical analysis focused on the first purpose. 


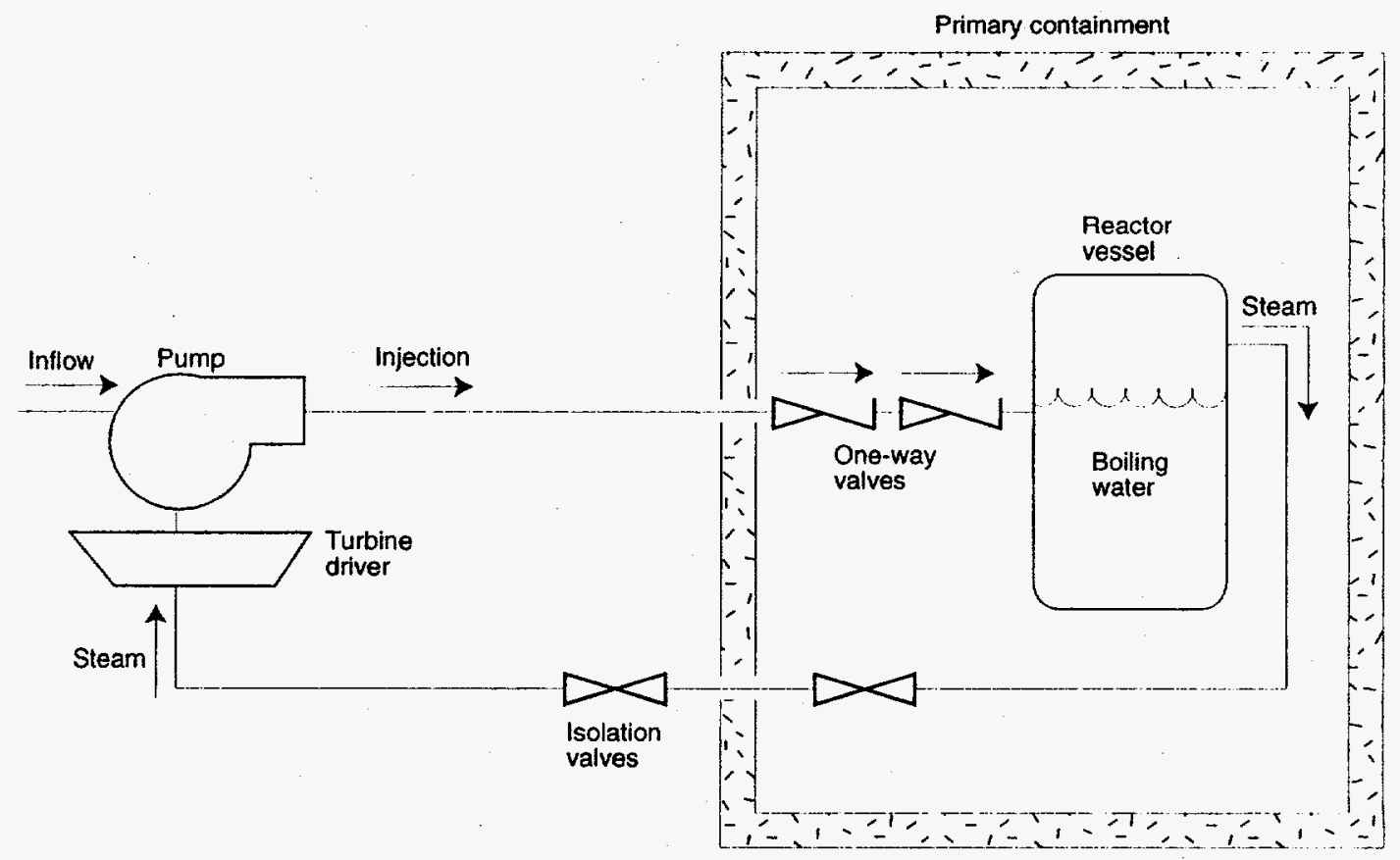

Figure 1. Extremely simplified diagram of RCIC system, showing isolation valves. Control valves, turbine exhaust line, instrumentation, and other hardware elements are not shown.

The injection pump is powered by a turbine, which is driven by steam from the reactor. Because the system contains piping through the primary containment, isolation valves are installed to prevent a loss of containment integrity in the event of a RCIC system pipe break outside the containment boundary. Closing either isolation valve maintains primary containment integrity, but it also prevents the system from performing its function of injection. During examination of data from past RCIC failures, two kinds of failures were noted that were initially hard to classify. (a) Spurious isolations occurred; that is, the isolation valves closed when they were not supposed to. (b) During full-system tests, the isolation valves would not close; therefore, the system was declared inoperable and manually isolated until the cause of the failure was repaired. The reason for manually isolating the system was to maintain primary containment integrity; because containment integrity takes precedence over the injection function of the RCIC system, the system must be taken out of service if the isolation valves cannot adequately provide for primary containment integrity.

Further thought into the purpose of the study convinced us that, for the statistical analysis, we were concerned only with the injection function of RCIC as modeled in a probabilistic risk assessment (PRA), the function of injecting water into the reactor vessel. Maintaining primary containment integrity was a concern for a different study. We classified events as hits, and called them "failures" in the study report, only if they prevented the injection safety function. We called the other events "inoperabilities," not failures. 
With this definition of hit, we considered the above two types of events: Spurious isolation would have prevented injection, but the valves were easily reopened in almost every case. When the isolation was only momentary, we did not count the event as a hit. Failure to isolate, on the other hand, did not prevent injection at all-steam was flowing to the turbine. Therefore, at the time of the test, we did not say that a hit (a failure that involved loss of the injection function) had occurred. Immediately after the failure to isolate, the system was taken out of service for repair. If a demand had then occurred, the system would have been out of service for maintenance, a failure mode in our classification system that would have been recorded as a hit. The failure to provide primary containment integrity, however, was not itself classified as a failure that involved loss of the system injection function.

Table 1. Event classification for reactor system studies.

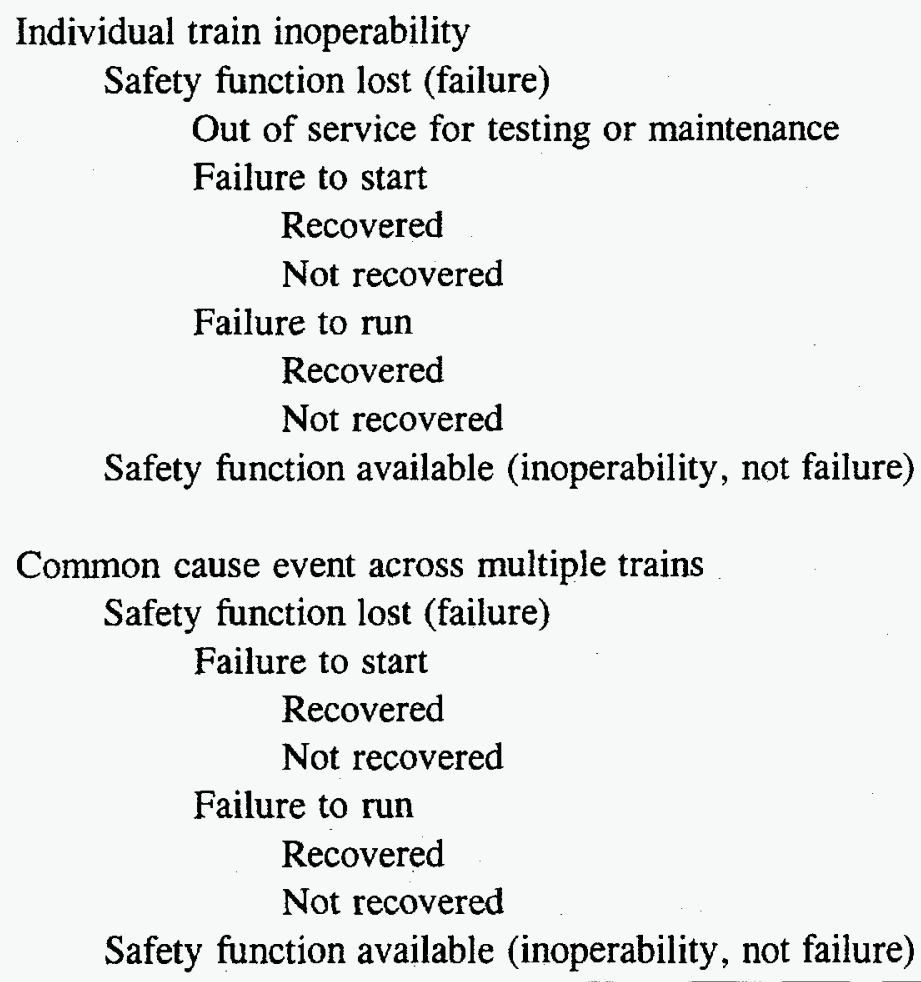

Table 1 shows a classification of failures and inoperabilities for a series of studies of nuclear power reactor safety systems; the RCIC study mentioned above was one study in this series. Indentations show subclassifications. For example, every train inoperability was either an event affecting a single train or a common cause event affecting multiple trains, not both. Within each such category, an event could be one of three kinds: failure to start, failure to run, or out of service for testing or maintenance. Note that out-of-service does not appear in the common cause portion of the table, because technical specifications prohibit taking out more than one train at a time for maintenance or testing while the reactor is at power. The failure modes were patterned after the failure modes in a PRA, because a purpose of the study was to compare the system data with PRA calculations. 
Depending on the data available for the system being studied, sometimes the failure modes had to be removed or combined. Single-train systems did not have the common cause portion of the classification. Often, too few common cause failures occurred to allow splitting them by failure mode, or too few failures of certain modes occurred to address recovery as a separate issue. Some of these decisions affected the coding (with certain fields not being coded), and some were made during the later statistical analysis. Nevertheless, Table 1 gave a useful framework, which could be modified in individual cases.

Table 2. Event classification for study of common cause failures.

\begin{tabular}{l|l|l|}
\hline \multicolumn{2}{c}{ Failure } & \multicolumn{2}{c}{ Command fault } \\
\cline { 2 - 3 } Individual & & \\
Nonlethal common cause & & \\
Lethal common cause & & \\
\cline { 2 - 3 } & &
\end{tabular}

Table 2 shows the classification that was used in a series of reports that analyzed common cause data using the binomial failure rate model. (See Atwood 1983.) Every event considered at all in the study was classified as either a failure or a command fault, and the analysis was performed in two ways, including and excluding command faults as hits. Every fault (that is, failure or command fault) was also classified as either an individual fault, a fault resulting from a nonlethal shock, or a fault resulting from a lethal shock.

\section{Demands}

Consider here failures on demand; failures in time are considered in the next subsection. Just as we spent considerable effort in clarifying the definition of a failure (or "hit"), we must now clarify the definition of a demand. A demand is an event that results in either a failure or a success. For statistical analysis, we further require

(1) The number of demands must be known, and of these demands, the number of failures and number of successes must be known.

Sometimes the word "known" is stretched, so that the number of demands is estimated, or so that the outcome of some demand is inferred from the event description. 
Operational failure data typically include both unplanned demands and various types of scheduled tests. If the unplanned demands are of greatest interest (regarded in some sense as "true" demands), and if the tests are to be counted as additional demands, then we require:

(2) The tests must be adequate substitutes for unplanned demands.

As an example, suppose that the observational unit under study is one of the pumps in the AFW system. Technical specifications require that when one pump is taken out of service for maintenance, the redundant pumps must first be tested to verify that they are operable. Assuming that the pump is started and run for a while, not simply started and immediately turned off, the study team may decide that the test is an adequate surrogate for an actual demand on the pump, so Requirement (2) is met. However, the number of such demands can be known only by detailed examination of the plant's maintenance records, and for studies that do not use maintenance records, Requirement (1) is not met - the total number of such demands is unknown. Without the number of demands, any such reported events cannot be used to estimate the probability of failure on demand.

As a second example, suppose that the observational unit of a study is the high pressure coolant injection (HPCI) system in a nuclear power plant. Suppose that a system failure is defined as any event when the system fails to perform its function of injecting water into the reactor vessel. The valves are tested every month. Suppose now that a critical valve fails during one of the monthly tests, so that the system is in a failed state. Can this failure be used to help estimate the probability of system failure on demand? Not directly, because Requirement (1) is not met: the total numbers of system successes on these monthly tests is unknown! Even if the valves all function correctly, we do not know whether the rest of the system would have failed if it had been tested. A failure of the valves is a system failure, but a success of the valves is not a system success; it is only a success of part of the system. In terms of Requirement (2), we must say that a valve test is not a demand on the whole observational unit, only a demand on part of the unit. To use such data, the HPCI system would have to be broken into smaller observational units (valves, pump, circuitry, etc.), connected by a logic model. Data would be collected for each unit, and the statistical analysis would result in an estimated failure probability for valves, a different estimated failure probability for the pump, etc. One could then use the logic model that shows how system failure is related to failure of the components, and obtain the estimated system failure probability by combining the component failure probabilities. This process is not unmanageable, and in fact large systems are frequently analyzed in terms of their pieces. Nevertheless, the example illustrates the complexity that arises from trying to divide the original observational unit (the HPCI system) by considering demands on a portion of the unit. 


\section{Exposure Time}

Consider now hits in time. The exposure time is defined as the time when hits could occur. For example, if a reactor system, such as HPCI, can only fail when the reactor is at power, the exposure time is the time when the reactor is at power. If a system is used both during power operations and during shutdowns, this usage sets the exposure time equal to the calendar time. This is the case for the low pressure coolant injection/residual heal removal (LPCI/RHR) pump in many nuclear power plants. However, the failure modes or failure rates may be different in the two plant operating modes, and the analyst should consider separate analyses for the two cases. Finally, some systems, such as electrical power systems in a nuclear power plant, are in use the same way at all times. For them, the exposure time coincides with the calendar time. 


\section{COUNTING HITS ACCURATELY}

The long discussion above dealt with definitions. Having, in principle, defined the events to count, we now consider how actually to count them. Two practical issues are considered here: completeness and correct coding.

\section{Completeness of Search}

Try very hard for a complete count of all the hits. It is definitely worth the effort, for these reasons:

- Undercounting the hits leads to an underestimate of the hit probability or the hit rate. This is anti-conservative.

- Sometimes the data source contains only a few hits. If a couple of them are overlooked, this omission can have a large effect on the conclusions of the statistical analysis.

- The study results will undergo review. A thorough reviewer sometimes comments, "This study left out my favorite event, ..." Any such omission should have a good reason, and not just be an oversight. Unjustified omissions bring the authors' credibility into question, perhaps doing long-term damage to their reputation with readers, the funder, or the funder's manager.

Therefore, do not naively assume that the first pass through the data sources will find all the hits. It will not. Think of all the different ways to sift the data to catch everything you are seeking. If narratives are searched electronically, look for the name of the observational unit both by its standard abbreviations and by its spelled-out name. Allow for the possibility of misspellings. If the unit is contained in one of two systems that are typically actuated together, or share equipment, look for mention of either system, even if the study is interested in only one. Because data bases have errors, try to find unrelated data bases that cover the same observational unit, and use each data base as a check on the other. If the observational unit has several failure modes, consider reviewing all the failure events even if you only want to count one failure mode, because sometimes failure modes are miscoded. Your attitude should be aggressive: "If it's out there, I am going to find it!" Keep notes of all the ways you look, and at the end write a section for the study report, describing all these quality assurance measures that were carried out to achieve a complete count of the hits. 
Some portions of a data set may not deserve examination. In planning the study, ignore a set of data unless all the hits are reported. For example, in a study of a safety system in a commercial nuclear power plant, suppose that a hit is defined as a failure of a train, and the system has several redundant trains, any one of which is sufficient for the system to perform its mission. A Licensee Event Report (LER) must be written if the entire system fails, or if an unplanned demand on a safety system occurs. Periodic tests, however, do not automatically generate LERs; if one train of the system fails but the others succeed, no report is written. Therefore, LER data from periodic tests should not be examined, because the failure count on such tests is incomplete.

Some analysts distrust any "voluntary" reporting, because they question its completeness. These analysts strongly prefer data that come from mandatory reporting, an examination of maintenance records, or some other source that is likely to be relatively complete.

\section{Correct Coding}

For correct coding, there is no substitute for having a clear understanding of the definitions. As mentioned above, during the beginning stages of a new project, when the definitions are still being formulated, a data collector can expect to go through the data sources several times, until the kinds of events in the data are understood and the definitions are correctly formulated. When this is achieved, the need for recoding the data will decrease sharply. Some recoding will still be necessary, but it will not stem from misunderstood definitions.

If judgment is required to interpret the data sources and create the data base, two data collectors are much better than one. It is very good to have two collectors, with each collector reviewing the work of the other, and the two collectors discussing the issues with a third person when they disagree. This internal peer review is a foundation for quality assurance.

The data collectors can speed up the whole process by not making decisions on the fly. Make provisional decisions and interpretations, but keep notes. The working copies of the data sources should look used, with notes written in the margins, phrases highlighted, and tabs stuck on. These notes are useful both early and late: (a) During the early data review, while the definitions are still being formulated, use your notes to identify similar events. Compare these events to each other, and discuss these events with all the other members of the study team. This seems slow, but in the long run it is faster to go through all that discussion early in the study, rather than later. Remember, one of the main reasons that definitions are neglected is that they seem obvious. The problem is that not everyone will 
initially agree on what is obvious. (b) Even after the definitions are well understood, the notes and highlights help the data collectors get to the main issues quickly, as they discuss differences of interpretation.

If the records consist of narratives, they will be difficult to interpret. If instead they are processed records in a data base, they will contain errors. In either case, therefore, try to compare your conclusions against other sources of data. For example, Licensee Event Reports were read to find failures of the HPCI system in nuclear power plants. For failures that occurred on periodic surveillance tests, the report narratives were used to determine whether the test was the kind performed once per operating cycle (approximately every 18 months) or the (less demanding) kind performed once every three months. When it became clear that the plants used different terminology for their tests, the interpretation of the narratives was questioned. Therefore, the event dates were compared to a data base that showed the operating history of each plant. With the understanding that the operating-cycle test would normally be performed immediately after a refueling outage, the data were recoded, changing about one fourth of the records. The point of this story is that the first data source initially appeared clear, but a check against a second data source provided important corrective information.

The situation is still more complicated if different independent data sources must be searched. For example, different plants have different means of keeping logs on component failure and maintenance events. Several sources may need to be consulted at a single plant, and similar sources may be called by different names at different plants. The best protection against the many possibilities for confusion is good communication: communication with the people closest to the data sources, who understand best what really happens at the plant; communication among the data collectors, for clear understanding of the definitions and for consensus judgment about interpretations; communication with the entire study team, for relevance of the data collected to the overall purposes of the project. For a project of this complexity, consider formalizing the communications by scheduling periodic meetings among all those who need to understand each other.

Finally, protect the data base against errors of data entry. Software that checks data fields for impossible values is very useful. A variation is to print out all the records (if any) for which some field does not have one of the allowed values; do this for each field.

In summary, the following techniques help ensure correct coding:

- clear understanding of the definitions,

- review of the data coding by another coder, 
- careful note-taking by the data collectors,

- comparison with other data sources,

- good communications, especially in complex projects,

- software tools to find errors in data entry.

As part of the quality assurance section of the final study report, explain the measures that were taken in your study to ensure accurate coding. 


\section{COUNTING OR ESTIMATING DEMANDS OR EXPOSURE TIME}

If demands are counted in a data set that is similar to that for hits, the advice for hits also applies to demands: take measures to count all the relevant demands and nothing but the relevant demands.

Often, however, the demands or exposure time must be obtained from a different type of source than the hit data base. For example, the number of demands may be estimated from the frequency of testing, or the exposure time may be approximated by the time in which the plant is in some operating mode. The data collector, the statistician, and the other members of the study team must talk with each other and see what can be achieved with the available information.

Do not assume, for example, that what is called a "monthly" test is performed once per month. Perhaps the same procedure is also used for every post-maintenance test, with the failure narratives not distinguishing between the two reasons for performing the test. Perhaps the test can only be performed when the plant is in a certain state. Perhaps one plant is subject to special rules, so that the test frequency there is different from that at all the other plants. Try to confirm even the most obvious assumptions by talking with people who are as close as can be found to the data sources.

If no reasonable estimate can be obtained for the demand count or the exposure time, statistical analysis has little value. The purpose of the study should be reconsidered. Therefore, the issue of how to obtain the demands or the exposure time should be resolved before an expensive investment of time is made in classifying and counting hits. 


\section{CHECKLIST}

The steps below are given in order, for most efficient use of manpower. Some reiteration through the topics will be necessary; for example, examination of the data may lead to reformulation of the earlier definitions. Nevertheless, no step should be postponed entirely in a rush to get to the next step.

The steps are directed to the whole study team. The data collection will be performed by the data collector(s), but most decisions should be considered by the whole team. The one requesting the study will provide input for Steps 1 and 2, and may also wish to be involved in Steps 3 through 5 .

1. What is the purpose of the study? Write this down before proceeding further.

2. What is the observational unit (system, component, etc.) being studied? Write down the definition, including the unit boundaries. If possible, find or create a diagram showing the unit and its boundaries.

3. What are the best available data sources for hits (typically, failures of the unit), consistent with the study's purpose?

4. How will the number of demands or the exposure time be determined? What are the best available data sources for demands or exposure times, consistent with the study's purpose?

5. How can the hits all be found? Think of as many ways as possible to search the available data sources. Brainstorm with the study team. If possible, try to search at least two data sources, letting each fill holes of the other.

6. How should all the events in the data source(s) be classified? Develop a classification system for the events in the data base(s), and decide which events will be used in the statistical analysis. Discuss this system with the study team. Review any events that were coded while the classification system was being developed, and discuss all questionable cases with the study team. State explicitly and discuss any decisions that were made while coding the data. Repeat this process until stability is achieved.

7. Reflect and review: After the data coding is underway, but before too much time has been spent, the data collectors should pass a portion of the data to the other members of the study team, for review. Discuss the meaning of everything in the data base, and make any necessary modifications. If the data contain no surprises that were missed by Step 6, little or no modification is needed. Nevertheless, it is wise to perform this review after everyone has had some time for reflection but before changing the data coding would be prohibitively difficult. 
8. Complete the data collection. At the end, the data collectors should write the report portion that describes the data collection and a quality assurance section that describes the measures taken to ensure complete counting and correct coding of the data. The data collectors should continue to consult with the data analysts as needed. 


\section{REFERENCES}

Atwood, Corwin L., 1983, Data Analysis Using the Binomial Failure Rate Common Cause Model, NUREG/CR-3437, EGG-2271.

Atwood, C. L., C. D. Gentillon, G. M. Grant, and M. B. Sattison, 1993, Reactor Core Isolation Cooling (RCIC) System Performance, 1987-1992 DRAFT, EGG-ERTP-10989.

Mosleh, Ali, 1986, "Hidden Sources of Uncertainty: Judgment in the Collection and Analysis of Data," Nuclear Engineering and Design, 93, pp. 187-198. 